\title{
Doença de Castleman axilar: Relato de Caso
}

\author{
Axillary Castleman's disease: Case Report \\ Enfermedad de Castleman axilar: Informe de Caso
}

Lorena Vieira Gusmão ${ }^{1 *}$, Paula Dandara Correia de Pinho1 ${ }^{1}$, Vanessa Olímpia Moura Guimarães Dota ${ }^{1}$, Érica Manuela da Silva Boa Sorte ${ }^{1}$, Luísa Malheiro Ferreira ${ }^{1}$, José Antônio Chaves de Aguiar Filho ${ }^{1}$, Gessandro Elpídio Fernandes Barbosa², Claudiojanes dos Reis ${ }^{3}$.

\section{RESUMO}

Objetivo: Relatar caso de doença de Castleman em região axilar, que se trata de um distúrbio linfoproliferativo raro, e revisar aspectos clínicos e terapêuticos da doença, enfatizando a importância do seu diagnóstico diferencial com patologias infecciosas e neoplásicas. Relato de caso: Trata-se de paciente assintomática apresentando nódulos mamário e axilar palpáveis, considerados suspeitos à ultrassonografia, sendo submetidos à core biopsy e punção aspirativa, respectivamente, apresentando-se benignos à microscopia. Realizada, todavia, exérese do nódulo axilar devido crescimento significativo ao exame clínico e ultrassonográfico após seis meses de acompanhamento, sendo o diagnóstico de doença de Castleman concluído após estudos histopatológico e imuno-histoquímico. Considerações finais: $O$ diagnóstico da referida doença, ainda que raro, deve ser sempre aventado diante de desordens linfoproliferativas, devido às semelhanças clínicas e radiológicas com variadas doenças infecciosas e malignas. A paciente apresentava a doença localizada, para a qual a exérese da lesão, fornece subsídio diagnóstico e representa o tratamento curativo.

Palavras-chave: Doença de Castleman, Tumor de Castleman, Transtornos linfoproliferativos, Linfadenopatia.

\begin{abstract}
Objective: To report a case of Castleman's disease in axillary area, which is a rare lymphoproliferative disorder, and to review clinical and therapeutic aspects of the disease, emphasizing the importance of its differential diagnosis with infectious and neoplastic pathologies. Case report: It is an asymptomatic patient with palpable mammary and axillary nodules, which are considered suspicious to the ultrasound, they were submitted to a core biopsy and aspiration puncture, respectively, and they were benign after the microscopy. However, an axillary nodule excision was performed due to significant growth on clinical and ultrasound examination after six months of medical monitoring, and the diagnosis of Castleman's disease was concluded after histopathological and immunohistochemical studies. Final considerations: Although rare, the diagnosis of this disease should always be made in the face of lymphoproliferative disorders, due to the clinical and radiological similarities with several infectious and malignant diseases. The patient had a localized disease, then the exeresis of the lesion provides a diagnostic support and represents a curative treatment.
\end{abstract}

Keywords: Castleman's Disease, Castleman Tumor, Lymphoproliferative disorders, Lymphadenopathy.

${ }^{1}$ Faculdades Unidades do Norte de Minas (FUNORTE), Montes Claros - Minas Gerais.

*E-mail: Iorena.gusmao@outlook.com

2Universidade Federal de Minas Gerais, Montes Claros - Minas Gerais.

3 Universidade Estadual de Montes Claros, Montes Claros - Minas Gerais.

SUBMETIDO EM: 9/2019

ACEITO EM: 10/2019

PUBLICADO EM: 1/2020

REAS/EJCH | Vol.12(1) | e1801 | DOI: https://doi.org/10.25248/reas.e1801.2020 Página 1 de 8 


\section{RESUMEN}

Objetivo: Informar el caso de la enfermedad de Castleman en la región axilar, que es un trastorno linfoproliferativo raro, y revisar los aspectos clínicos y terapéuticos de la enfermedad, destacando la importancia de su diagnóstico diferencial com patologías infecciosas y neoplásicas. Informe de caso: Una paciente asintomática con nódulos palpables mamarios e axilares, considerada sospechosa en la ecografía, sometida a biopsia central y punción por aspiración, respectivamente, y benigna bajo microscopía. Sin embargo, la escisión del nódulo axilar se realizo debido a um crecimiento significativo em el examen clínico y ecográfico después de estudios histopatológicos e inmunohistoquímicos. Consideraciones finales: Aunque es raro, el diagnóstico de esta enfermedad siempre debe hacerse frente a los trastornos linfoproliferativos, debido a las similitudes clínicas y radiológicas con diversas enfermidades infecciosas y malignas. El paciente tenía una enfermedad localizada, para la cual la escisión de la lesión proporciona soporte diagnóstico y representa un tratamento curativo.

Palabras clave: Enfermedad de Castleman, Tumor de Castleman, Trastornos linfoproliferativos, Linfadenopatía.

\section{INTRODUÇÃO}

A doença de Castleman (DC) é uma desordem linfoproliferativa rara, descrita pela primeira vez pelo patologista Benjamin Castleman, em 1956, como "hiperplasia hialinizante linfoide". Pode ainda ser denominada hiperplasia angiofolicular, hamartoma linfoide, linfoma gigante benigno, linforeticuloma folicular e angiomatose linfoide, refletindo a relativa incerteza quanto à sua origem e tipificação como tumor ou doença inflamatória infecciosa (YEH CM, et al., 2007).

A prevalência não está bem estabelecida na população em geral, tendo poucos casos relatados no Brasil. Estima-se que nos Estados Unidos haja entre 30.000-100.000 casos, baseado no número de pacientes com linfadenomegalias atendidos em centros oncológicos e que posteriormente foram diagnosticados como DC (MUHSEIN KA, et al., 2004).

Sua etiologia e patogênese são pouco elucidadas, mas acredita-se haver hiperestimulação antigênica induzida por vírus de forma crônica e mediada por interleucina 6, estando alguns casos ligados ao herpes vírus 8 (HHV-8), vírus da imunodeficiência humana (HIV) e Epstein-Barr (EBV) (CASPER C, 2005; BLANCO EC, et al., 2007; MUHSEIN KA, et al., 2004).

Apesar de seu caráter benigno à histologia, a DC pode manifestar-se agressivamente ou associar-se a outros distúrbios como linfoma e sarcoma de Kaposi. (KRAWCZUN GA, et al., 2007). O mediastino é a localização mais frequente da doença, porém outros sítios podem ser acometidos, como pescoço, pelve e, raramente, axila, além disso, não há predominância de sexo ou idade. (VALLE L, et al., 2006; GIDVANIVK, et al., 2001).

Apresenta duas formas clínicas: unicêntrica (mais comum) e multicêntrica, com envolvimento de múltiplos órgãos e sintomas sistêmicos; e ainda dois subtipos patológicos: hialino vascular e plasmocítico (ANDRADE NA, et al., 2014).

A DC unicêntrica, mais encontrada em adultos jovens, manifesta-se sob a presença de massa em mediastino, pescoço, ou menos comumente, em abdome. É incomum que haja sintomas nesta apresentação, mas quando presentes, são gerados pelo efeito de massa da lesão. Já a forma multicêntrica cursa com polilinfadenopatia e, frequentemente, há envolvimento de múltiplos órgãos e sintomas sistêmicos. Costuma ocorrer na sexta década de vida e possui uma história natural mais agressiva (SANZ GARCIA, et al., 1999; WATERSON A e BOWER M, 2004; GIDVANI VK, et al., 2001).

O objetivo deste estudo foi relatar um caso de DC de localização axilar, pouco comum, e revisar aspectos clínicos e terapêuticos desta doença, enfatizando a importância do diagnóstico diferencial com doenças neoplásicas e infecciosas. 


\section{DETALHAMENTO DO CASO}

Paciente feminina, 33 anos, atendida em consultório de mastologia, encaminhada pelo serviço de atenção primária de sua cidade, com relato de nódulo palpável em mama esquerda, percebido há seis anos, sem crescimento significativo e com resultado de ultrassonografia evidenciando imagem nodular hipoecogênica apontada pelas setas como área mais escura em relação ao restante do tecido - e de formato ovóide, na união de quadrantes laterais da mama esquerda, paralela à pele, de margens circunscritas, multilobulada, de aproximadamente 2,6 $\mathrm{cm}^{3}$ (Figura 1); e ainda, imagem nodular, também hipoecogênica, no prolongamento axilar da mama direita, ovoide, paralela à pele, sem acústica posterior, de margens circunscritas delimitadas pelas setas, de aproximadamente $5,8 \mathrm{~cm}^{3}$ (Figura 2).

Figura 1 - Ultrassonografia da mama esquerda evidenciando nódulo hipoecogênico e multilobulado na união dos quadrantes laterais.

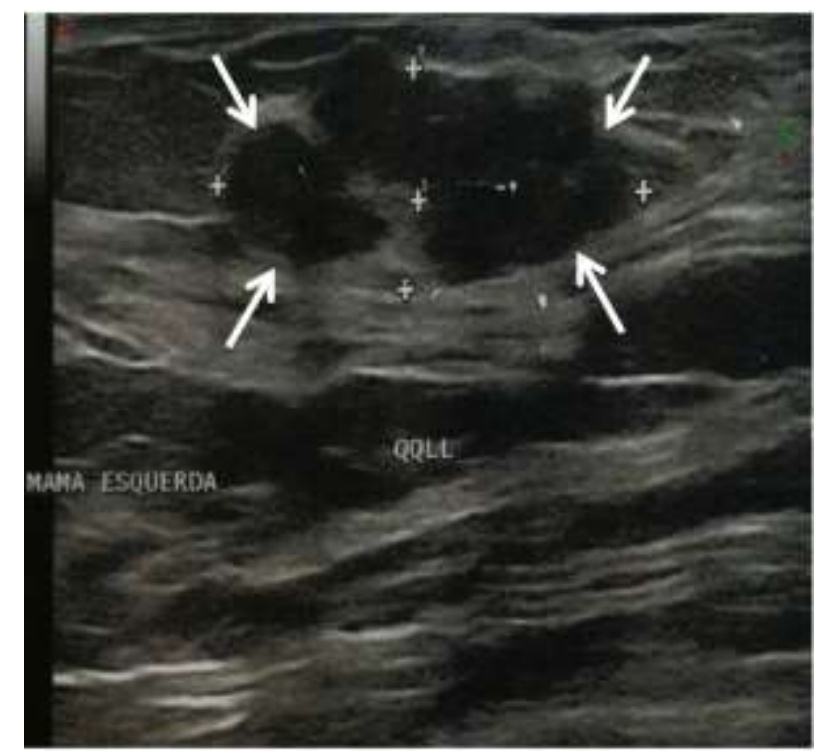

Fonte: Gusmão LV, et al., 2019.

Figura 2 - Ultrassonografia da mama direita demonstrando nódulo hipoecogênico no prolongamento axilar.

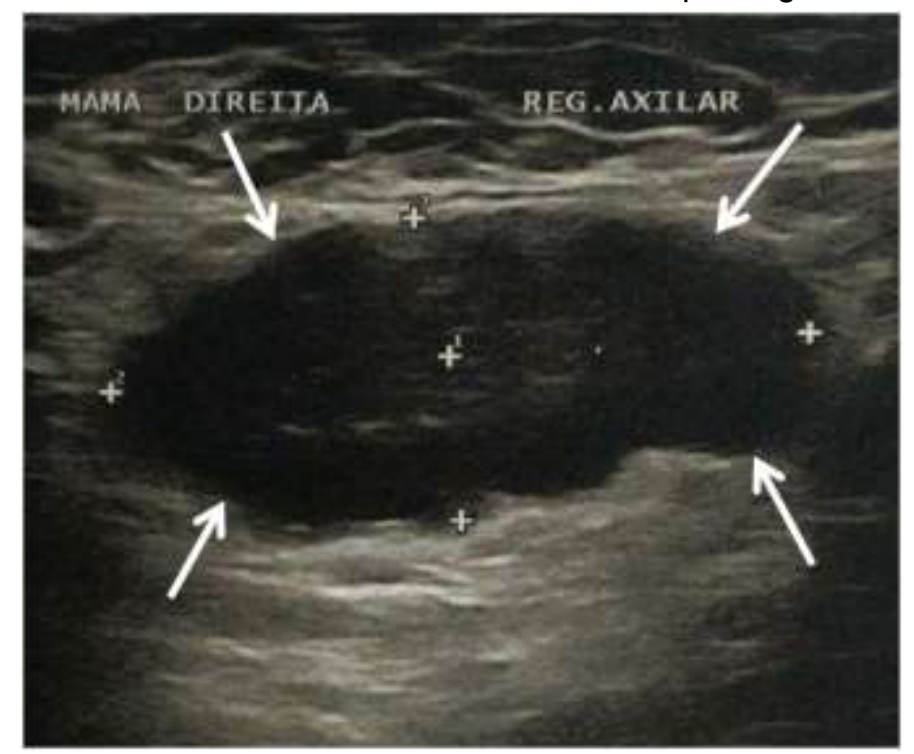

Fonte: Gusmão LV, et al., 2019. 
A paciente negava comorbidades, uso de medicação contínua e contracepção hormonal. Referia três gestações, partos vaginais sem intercorrências e lactações fisiológicas. Menarca aos 14 anos e telarca aos 12. Ciclos menstruais regulares de 28 dias, fluxo moderado, com duração média de quatro dias. Não possuía história familiar de câncer.

Ao exame físico, encontrava-se em bom estado geral, orientada, corada, hidratada, eupneica, afebril, normotensa. Exames dos aparelhos respiratório, digestório e cardiovascular sem alterações. Mamas simétricas e pendulares, sem abaulamentos ou retrações, com um nódulo palpável na união dos quadrantes laterais da mama esquerda, de consistência fibroelástica, medindo aproximadamente $2,5 \times 1 \mathrm{~cm}$ e outro no prolongamento axilar da mama direita, de cerca de $2 \times 3 \mathrm{~cm}$ com consistência fibroelástica. Ausência de descarga papilar. Linfonodos supraclaviculares não palpáveis.

Devido à classificação dos achados descritos em ultrassonografia previamente realizada pela paciente, como pertencentes à categoria 4 (suspeito de malignidade) no sistema de relatório de dados sobre imagem da mama denominado Breast Imaging Reporting and Data System (BIRADS), seguiu-se a propedêutica a fim de elucidar a natureza dos nódulos. O nódulo em mama esquerda foi submetido a core biopsy devido à melhor acurácia deste método para formações sólidas. Já o nódulo do prolongamento axilar da mama direita, por apresentação radiológica e localização sugestivas de linfonodomegalia, foi submetido a punção aspirativa por agulha fina (PAAF).

Ao exame histopatológico, os fragmentos coletados por core biopsy apresentavam-se compatíveis com fibroadenoma. Enquanto o material da PAAF da mama direita, submetido à análise citopatológica, mostravase altamente vascularizado, continha numerosos linfócitos maduros e alguns histiócitos, não apresentando células neoplásicas. Diante da natureza benigna dos achados, optou-se por prosseguir o acompanhamento ambulatorial através de reavaliação após seis meses.

Decorrido este tempo, retornou à consulta sem queixas. O exame clínico das mamas, no entanto, evidenciou aumento do nódulo do prolongamento axilar direito para aproximadamente $3,5 \times 3 \mathrm{~cm}$. À ultrassonografia, confirmou-se o crescimento do nódulo sólido e vascularizado no prolongamento axilar direito para $10,9 \mathrm{~cm}^{3}$ - anteriormente de $5,8 \mathrm{~cm}^{3}$-, delimitado pelas setas em ambas as imagens (Figura 3A e 3B); e linfonodomegalia axilar ipsilateral. O nódulo da mama esquerda apresentou-se idêntico ao exame clínico e ultrassonográfico anterior.

Figura 3A e 3B - Ultrassonografia da mama direita evidenciando nódulo sólido no prolongamento axilar.

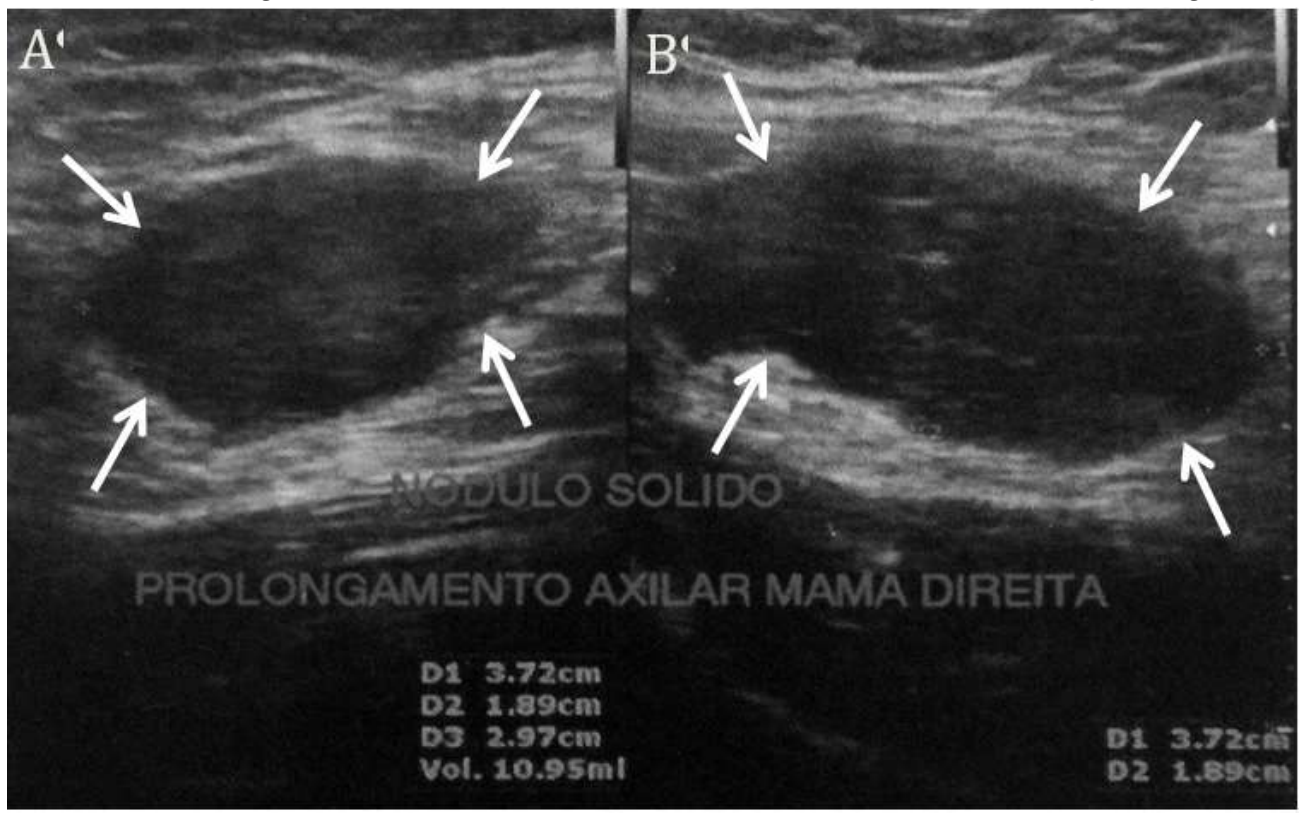

Fonte: Gusmão LV, et al., 2019. 
Considerando o crescimento significativo do nódulo em mama direita, foi indicada exérese do mesmo. A paciente apresentou exames laboratoriais pré-operatórios dentro dos valores de referência, o que corrobora com a apresentação clínica restrita ao tecido linfático. Hemácias: 4,4 milhões $/ \mathrm{mm}^{3}$; hemoglobina: $13,4 \mathrm{~g} / \mathrm{dL}$; hematócrito: $44 \%$; leucócitos: $6.499 / \mathrm{mm}^{3}$; plaquetas: $251.000 / \mathrm{mm}^{3}$; ureia: $22 \mathrm{mg} / \mathrm{dL}$; creatinina: $0,5 \mathrm{mg} / \mathrm{dL}$; glicemia: $92 \mathrm{mg} / \mathrm{dL}$; tempo de protrombina: 12,8 segundos; atividade de protrombina: $92 \%$; RNI: 1,03.

A excisão cirúrgica foi realizada sem intercorrências, através de incisão da pele e tecido subcutâneo, identificação e retirada do nódulo, seguidas de fechamento com sutura por planos. O material, tecido pardoamarelado, irregular, macio e untuoso, foi enviado para análise histopatológica.

À microscopia, foi identificado linfonodo de arquitetura parcialmente subvertida devido à presença de folículos com centros germinativos involuídos, contendo depósitos hialinos, penetrados por vênulas hialinizadas e circundados por anéis de linfócitos pequenos. A zona interfolicular apresentava densa população de linfócitos, plasmócitos, vasos hialinizados e espessas traves fibrosas de permeio. Os achados foram sugestivos de Doença de Castleman.

A fim de complementar a propedêutica, foi realizado estudo imuno-histoquímico, que revelou presença de população linfoide mista reacional com positividade para marcadores de linfócitos $B$ e $T$, sustentando o diagnóstico de DC na sua variante hialino-vascular.

Foram solicitadas tomografias computadorizadas de tórax e abdome para diferenciação do caso entre doença unicêntrica ou multicêntrica. Não sendo evidenciados outros focos da doença através destes exames, concluiu-se que se tratava de DC unicêntrica de localização axilar.

A paciente seguiu em acompanhamento clínico ambulatorial, permanecendo assintomática, sem recidiva da doença após a exérese cirúrgica. Após isso, nenhum tratamento complementar foi instituído.

\section{DISCUSSÃO}

A Doença de Castleman é uma causa rara de proliferação do tecido linfoide, com etiologia e patogênese ainda pouco esclarecidas, apesar de evidências apontarem para uma possível relação da doença com infecções virais. No caso abordado, não foram identificadas condições clínicas que suscitassem a hipótese de co-infecção (CASPER C, 2005; BLANCO EC, et al., 2007; DHAM A e PETERSON BA, 2007).

Existem duas formas de síndrome clínica: unicêntrica (90\%) e multicêntrica (10\%); e dois subtipos patológicos: hialino vascular (80-90\%) e plasmocítico (10-20\%), sendo que alguns casos são classificados como padrão misto já que apresentam elementos comuns aos dois subtipos (2\%) (ANDRADE NA, et al., 2014).

A localização mediastino-pulmonar corresponde a $70 \%$ dos casos e atinge preferencialmente mediastino ântero-superior. As formas abdominais (10\%) são de grande tamanho, em geral, e localizam-se na região mesentérica ou retroperitoneal. Foram ainda descritas outras localizações anatômicas, incluindo pescoço (14\%), pelve (4\%) e axila (2\%) (VALLE L, et al., 2006; PINHEIRO VGF, et al., 2008).

O caso relatado destaca-se pelo sítio pouco comum de acometimento. A linfonodomegalia axilar isolada, por suscitar tantos outros diagnósticos mais comuns, faz com que a DC não esteja entre as principais hipóteses aventadas na prática médica diária. Chamamos atenção, com a apresentação deste relato, para a importância de se inserir a DC no diagnóstico diferencial de doenças linfoproliferativas.

Quanto aos aspectos histológicos, a variante hialino-vascular dispõe-se de pequenos centros foliculares envoltos por tecido hialino e com intensa vascularização entre os folículos. Há descrição da zona do manto com conformação semelhante à "casca de cebola", quando circundada por camadas linfocíticas concêntricas. A variante plamocítica, por sua vez, caracteriza-se por menor vascularização e grandes folículos hiperplásicos dispostos em meio a múltiplas células plasmáticas. A forma mista possui aspecto intermediário entre estas apresentações. (SOUZA LJ, et al., 2005; CASPER C, 2005; SCHUNEMANN E, et al., 2000; IZUCHUKWU IS, et al., 2003). 
As variedades plasmocítica e multicêntrica são as que mais comumente se associam a processos malignos, bem como a co-infecções virais (ANDRADE NA, et al., 2014).

$\mathrm{O}$ caso descrito tratava-se de doença unicêntrica, visto que a paciente apresentava linfadenopatia localizada, sem manifestações sistêmicas ou alterações laboratoriais. $\mathrm{O}$ achado histológico do subtipo hialino vascular corroborou para esta classificação, já que é observado em $90 \%$ das DC unicêntricas. O diagnóstico definitivo depende da análise histopatológica da lesão após excisão cirúrgica, e pode ser complementado por análise imuno-histoquímica em casos duvidosos. A PAAF somente deve ser considerada se indisponibilidade cirúrgica devido às altas taxas de erro de amostragem (SANZ GARCIA RM, et al., 1999; GIDVANI VK, et al., 2001; DHAM A, PETERSON BA, 2007; ANDRADE NA, et al., 2014; BLANCO EC, et al., 2007;).

Pela apresentação pouco específica, sob a forma unicêntrica, com uma adenopatia localizada sem sintomas gerais ou alterações laboratoriais, a DC não estava entre as primeiras hipóteses diagnósticas consideradas, não se seguindo, portanto, a propedêutica recomendada pela literatura. Inicialmente, foi solicitada análise histopatológica das lesões através da core biopsy, sugerindo fibroadenoma - tumor fibroepitelial benigno da mama, caracterizado por proliferação de elementos estromais e glandulares -, e estudo citopatológico a partir da PAAF evidenciando alterações inespecíficas benignas. Diante da natureza dos achados, prosseguiu-se com acompanhamento ambulatorial que permitiu identificar crescimento do nódulo em prolongamento axilar direito, submetido então a exérese para análise histopatológica. À microscopia, foram reveladas alterações compatíveis com doença de Castleman, sendo realizado estudo imuno-histoquímico que fornece melhor acurácia diagnóstica.

Os métodos de imagem, como tomografia computadorizada (TC) e ressonância magnética (RM), são úteis para identificação de doença multicêntrica, bem como para planejamento terapêutico (CASPER C, 2005).

A Doença de Castleman na TC costuma apresentar-se como uma massa homogênea, bem delimitada, de realce moderado a intenso, com o tipo hialino vascular tendendo a um realce mais evidente devido maior vascularização quando comparado ao tipo plasmocítico. A acentuada captação de contraste, que geralmente não é observada nos linfomas, pode auxiliar no diagnóstico diferencial dessas patologias. A RM habitualmente revela massa hipointensa em T1 e hiperintensa em T2 (YEH CM, et al., 2007; ANDRADE NA, et AC., 2014; BRITO AJP, 2009). A paciente não apresentava evidências clínicas de acometimento sistêmico da doença, sendo confirmada, com TC de tórax e abdome, a inexistência de outros focos de DC.

O diagnóstico diferencial da DC inclui tumores como o linfoma, afecções que cursam com linfonodomegalia, como as doenças inflamatórias (tuberculose, sarcoidose etc.); infecção pelo vírus HIV e doenças autoimunes (MUHSEIN KA, et al., 2004; YEH CM, et al., 2007).

O aumento isolado do linfonodo axilar é importante preditor de neoplasia, sendo os linfomas e as metástases de cânceres que acometem a parede torácica, como o de mama, diagnósticos diferenciais relevantes nesses casos. A suspeita de câncer aumenta a depender da idade do indivíduo, sendo que aqueles com mais de 40 anos apresentam risco 10 vezes maior. Quanto ao tamanho, linfonodos menores de $1 \mathrm{~cm}^{2}$ representam risco baixo para doenças neoplásicas, com aumento considerável do risco a partir de $2,25 \mathrm{~cm}^{2}$ (FIJTEN GH e BLIJHAM GH, 1998; PANGALIS GA, 1993).

Os linfomas geralmente se apresentam como linfonodomegalia indolor, e pode não haver qualquer outro sintoma sistêmico associado, fazendo-se necessária análise microscópica de lesão, devido clínica comum a DC e outras doenças. Todavia, podem acometer órgãos contíguos, trazendo múltiplos sinais e sintomas, devendo também ser diferenciados da DC, nesse caso, sob sua forma multicêntrica (DIDIER NETO FMFeKISO KM, 2013).

Nas metástases, os linfonodos apresentam consistência mais endurecida, são mal delimitados, aderentes a planos profundos e pouco móveis, diferentemente do observado na paciente deste relato. Entretanto, devido à presença de nódulo mamário concomitante à linfonodomegalia, a hipótese de câncer de mama com metástase linfonodal também foi aventada, ainda que não houvesse comprometimento sistêmico esperado para esse estágio da doença neoplásica (DIDIER NETO FMF e KISO KM, 2013). 
As doenças infecciosas também podem cursar com linfadenomegalia, geralmente acompanhada de febre, sendo o aumento do gânglio localizado ou generalizado. Em patologias como a tuberculose e a paracoccidioidomicose, o microorganismo pode estar presente no gânglio, enquanto na síndrome da imunodeficiência adquirida (AIDS) na mononucleose infecciosa, seu aumento decorre da resposta imune, sem a presença do agente patológico em seu interior. A alteração ganglionar pode ser prolongada ou curta (ZAGO MA, 2013).

A exérese da lesão compreende o tratamento curativo para a doença unicêntrica, especialmente no subtipo hialino-vascular. No caso da variante plasmocítica, pode ser necessário tratamento complementar com corticoterapia e/ou quimioterapia para controle sintomático adequado. Pacientes com contraindicação à cirurgia têm a radioterapia como opção terapêutica, com resposta incerta em ambos os tipos histológicos. Entretanto, a literatura carece de mais estudos comparativos entre as modalidades de tratamento (CASPER C, 2005; BLANCO EC, et al., 2007; BERGE MVD, et AC., 2002).

Para a forma multicêntrica, não há consenso quanto à melhor abordagem terapêutica. Tem sido proposto o uso da corticoterapia isolada, quimioterapia com vincristina, ciclofosfamida e doxorrubicina ou associação de ambas. Outras opções mais recentes incluem o interferon-alfa e a talidomida, com resultados controversos. Antivirais podem ser utilizados em pacientes com doença multicêntrica e infecção por HHV 8. A radioterapia não tem obtido resultados favoráveis (JONGSMA TEF,et al., 2007; CAPRIO JS, et al., 2016;SOUZA LJ, et al., 2005; IZUCHUKWU IS, et al., 2003; CORBELLINO M, et al., 2001).

A DC unicêntrica apresenta regressão total na grande maioria dos casos submetidos ao tratamento cirúrgico. Já na doença multicêntrica, as alternativas disponíveis determinam respostas clínicas variáveis, mantendo prognóstico reservado pelo potencial risco de associar-se a outras doenças graves (CASPER C, 2005; CORBELLINO M, et al., 2001).

A paciente foi submetida à exérese cirúrgica, sem intercorrências, mantendo-se assintomática durante acompanhamento clínico ambulatorial. Não foram identificados novos sítios da doença, não sendo empregado nenhum tratamento complementar.

\section{CONSIDERAÇÕES FINAIS}

O estudo evidencia o relato de caso de paciente com DC de localização axilar, pouco comum, submetida a exerése da lesão como tratamento e apresentando boa evolução. Embora rara, a possibilidade de Doença de Castleman deve ser aventada em todos os casos de linfadenopatia. Ressalta-se que o diagnóstico clínico e anatomopatológico pode ser difícil e inconclusivo, sendo necessário a realização de estudo imunohistoquímico. A paciente apresentava DC de localização axilar, unicêntrica, na sua variante hialino-vascular, para a qual a exérese da lesão, além de fornecer subsídio diagnóstico, representa o tratamento curativo. Entretanto, na forma multicêntrica, persistem controvérsias no que diz respeito ao tratamento. O retardo em se instituir a terapêutica apropriada no caso em questão, reforça a necessidade de se inserir a DC no diagnóstico diferencial de doenças linfoproliferativas.

\section{REFERÊNCIAS}

1. ANDRADE NA, et al. Doença de Castleman Cervical: relato de três casos. Revista da AMRIGS. 2014; 58(1):61-64.

2. BERGE MVD, et al. Hyaline Vascular Castleman's disease: a case report and brief review of the literature. Netherlands Journal of Medicine. 2002; 60(11):444.

3. BLANCO EC, et al. Castleman's disease of the neck. Acta Otorrinolaringol. Esp. 2007; 58(9):440-4.

4. BRITO AJP, et al. Doença de Castleman. Rev. Bras. Cir. Cabeça Pescoço. 2009; 38(2):120-1.

5. CAPRIO JS, et al. Doença de Castleman: relato de caso. Rev. Soc. Bras. Clin. Med. 2016; 14(3):163-5.

6. CASPER C. The etiology and management of Castleman disease at 50 years: translating pathophysiology to patient care. Br J Haematol. 2005; 129(1):3-17.

7. CORBELLINO M, et al. Long-term remission of Kaposi sarcoma-associated herpesvirus-related multicentric Castleman disease with anti-CD20 monoclonal antibody therapy. Blood2001; 98(12):3473-5. 
8. DIDIER NETO FMF, KISO KM. Comprometimento dos linfonodos em adultos. Arq. Med. Hosp. Fac. Cienc. Med. Santa Casa São Paulo. 2013; 58:79-87.

9. DHAM A, PETERSON BA. Castleman disease. Current Opinion in Hematology. 2007; 14:354-9.

10. ERKAN N, et al. Peripancreatic Castleman disease. JOP. 2004; 5(6):491-4.

11. FIJTEN GH, BLIJHAM GH. Unexplained lymphadenopathy in family practice. An evaluat of the probability of malignant causes and the effectiveness of physician's workup. J Fam. Pract. 1988; 27:373-6.

12. GIDVANI VK, et al. Castleman's disease: atypical manifestation in an 11-year-old girl. South Med J. 2001; 94(2): 2503.

13. IZUCHUKWU IS, et al. Anunusual presentation of Castleman's disease: a case report. BMC Infect. Dis. 2003; 3(20).

14. JONGSMA TEF, et al. Castleman's disease: a rare lymphoproliferative disorder. European Journal of Medicine 2007; 18:87-9.

15. KRAWCZUN GA, et al. Castleman's disease orangio follicular hyperplasia as a solitary pulmonary nodule: case report. J Bras. Pneumol. 2007; 33(2):226-8.

16. MUHSEIN KA, et al. Localized Castleman's disease presenting as a vascular right iliac fossa mass. Asian $\mathrm{J}$ Surg. 2004; 27(1):54-7.

17. PANGALIS GA, et al. Clinical approach to lymphadenopathy. Semin. Oncol. 1993; 20:570-82.

18. PINHEIRO VGF, et al. Doença de Castleman associada a derrame pleural. J Bras. Pneumol. 2008; 34(8):626-630.

19. SANZ GARCIA RM, et al. Enfermedad de Castleman localizada associada a linfoma de alto grado. AnMed Interna. 1999; 16(6):305-7.

20. SCHUNEMANN E,et al. Doença de Castleman retroperitoneal - relato de um caso e revisão da literatura. Acta Oncol. Bras. 2000; 20.

21. SOUZA LJ,et al. Doença de Castleman multicêntrica. Rev. Soc. Bra. Clín. Méd. 2005; 3(1):20-23.

22. VALLE L, et al. Doença de Castleman retroperitoneal caso clínico e revisão da literatura. Arquivos de Medicina. 2006; 19(1-2): 29-33.

23. WATERSON A, BOWER M. Fifty years of multicentric Castlemans disease. Acta Oncol. 2004; 43(8):698-704.

24. YEH CM, et al. Castleman's disease mimicking intrapulmonary malignancy. Ann Thorac. Surg. 2007; 84(2):6-7.

25. ZAGO MA, et al. Tratado de hematologia. São Paulo: Atheneu; 2013.78p. 